\title{
Gravity induced wave function collapse
}

\author{
G. Gasbarri, ${ }^{1,2,3}$ M. Toroš ${ }^{2,3}$ S. Donadi, ${ }^{2,3}$ and A. Bassi ${ }^{2,3}$ \\ ${ }^{1}$ Abdus Salam ICTP, Strada Costiera 11, 34151 Trieste, Italy \\ ${ }^{2}$ Department of Physics, University of Trieste, Strada Costiera 11, 34151 Trieste, Italy \\ ${ }^{3}$ Istituto Nazionale di Fisica Nucleare, Trieste Section, Via Valerio 2, 34127 Trieste, Italy
}

(Received 17 July 2017; published 13 November 2017)

\begin{abstract}
Starting from an idea of S. L. Adler [in Quantum Nonlocality and Reality: 50 Years of Bell's Theorem, edited by M. Bell and S. Gao (Cambridge University Press, Cambridge, England 2016)], we develop a novel model of gravity induced spontaneous wave function collapse. The collapse is driven by complex stochastic fluctuations of the spacetime metric. After deriving the fundamental equations, we prove the collapse and amplification mechanism, the two most important features of a consistent collapse model. Under reasonable simplifying assumptions, we constrain the strength $\xi$ of the complex metric fluctuations with available experimental data. We show that $\xi \geq 10^{-26}$ in order for the model to guarantee classicality of macro-objects, and at the same time $\xi \leq 10^{-20}$ in order not to contradict experimental evidence. As a comparison, in the recent discovery of gravitational waves in the frequency range 35 to $250 \mathrm{~Hz}$, the (real) metric fluctuations reach a peak of $\xi \sim 10^{-21}$.
\end{abstract}

DOI: $10.1103 /$ PhysRevD.96.104013

\section{INTRODUCTION}

The possibility for quantum mechanics to be the limiting case of an underlying nonlinear theory has been often considered in the literature [1-6]. A straightforward motivation is that linear models typically are an approximation of nonlinear ones [5]. A stronger motivation is that they open the way to solving the quantum measurement problem [7]. In this latter context, models of spontaneous wave function collapse [8-11] provide a consistent phenomenology describing the collapse of the wave function during a measurement via extra nonlinear and stochastic terms added to the dynamics. Due to their intrinsic nonlinearity, these models also offer a way out for some of the puzzles in quantum gravity and cosmology [12-14].

The common feature of all collapse models is a classical noise, coupled nonlinearly to the quantum wave function. The typical collapse equation, in the Itô form, is

$$
\begin{aligned}
d \psi_{t}= & {\left[-\frac{i}{\hbar} \hat{H}_{0} d t+\sqrt{\lambda} \sum_{j}\left(\hat{A}_{j}-\left\langle\hat{A}_{j}\right\rangle_{t}\right) d W_{j, t}\right.} \\
& \left.-\frac{\lambda}{2} \sum_{j}\left(\hat{A_{j}}-\left\langle\hat{A_{j}}\right\rangle_{t}\right)^{2} d t\right] \psi_{t},
\end{aligned}
$$

where $\hat{H}_{0}$ is the standard quantum Hamiltonian, $\left\{\hat{A}_{j}\right\}_{j}$ is a set of self-adjoint commuting operators, and $\left\langle\hat{A}_{j}\right\rangle_{t}=\left\langle\psi_{t}\left|\hat{A}_{j}\right| \psi_{t}\right\rangle$ and $W_{j, t}$ are a set of independent Wiener processes, which force the wave function to collapse towards one of the common eigenstates of the operators $\hat{A}_{j}$ [15]. The positive coupling constant $\lambda$ sets the strength of the collapse mechanism.

Equation (1) should be considered as a phenomenological equation, raising the question of why it takes that form. A justification comes from the following argument first proposed by Adler [6]. Consider the Hamiltonian

$$
\hat{H}=\hat{H}_{0}+i \hbar \sqrt{\lambda} \sum_{j} \hat{A}_{j} w_{j, t}
$$

where $w_{j, t}=d W_{j, t} / d t$ is a set of independent white noises. It describes the coupling of a quantum system with external classical noises through the operators $\hat{A}_{j}$. It is a reasonable phenomenological ansatz, except for the fact that the second term is anti-Hermitian [16]. As a consequence, the norm of $\psi_{t}$ is not conserved, jeopardizing the physical meaning of the wave function. The obvious thing to do is to replace $\psi_{t}$ with $\psi_{t} /\left\|\psi_{t}\right\|$, but this brings in a serious problem: the resulting equation is nonlinear, and also the stochastic ensemble of states evolves nonlinearly, even in the average. This leads to superluminal signaling [21]. The problem can be avoided if one adds extra terms in Eq. (2), such that the master equation for density matrix $\rho_{t}=$ $\mathbb{E}\left[\left|\psi_{t}\right\rangle\left\langle\psi_{t}\right|\right]$ associated with the ensemble becomes linear (and of the Lindblad type [22-24]). These new terms are precisely those which lead to Eq. (1). Appendix A contains the derivation of what is outlined here.

In the sense explained above, the requirements of norm conservation and no superluminal signaling added to Eq. (2) give the desired collapse equation. The hope is that a sensible nonlinear prequantum theory, which leads to a dynamics for the wave function at the phenomenological level, will naturally embody both requirements. The open issue now is how to justify $\hat{H}$ in (2), in particular, why the coupling should be anti-Hermitian, and what the suitable choice is for the operators $\hat{A}_{j}$, which select the basis along which the collapse occurs. While there is no answer to the first question-at least no more than the hope that the prequantum theory will provide a natural answer-one can say more about the second question. 
Quite often the literature suggests that the collapse is driven by gravity [25-32]. This is the only possibility one can have, to link the collapse to a known force, since all other forces as we know them have been successfully quantized; therefore, they cannot provide the anti-Hermitian coupling needed for the nonlinear collapse. But there is a stronger motivation. The collapse scales with the mass/size of the system $[8,9]$ and localizes the wave function in space. Then, the natural candidate for the operators $\hat{A}_{j}$ is the local mass density $\hat{m}(\mathbf{x})=\sum_{i} m_{i} \delta^{(3)}\left(\mathbf{x}-\hat{\mathbf{x}}_{i}\right)$, coupled to a noise $w(\mathbf{x}, t)$ spread through space [33],

$$
\hat{H}=\hat{H}_{0}+i \hbar \sqrt{\xi} \int d^{3} x \hat{m}(\mathbf{x}) w(\mathbf{x}, t) .
$$

A random gravitational field naturally provides such a coupling (see Appendix B), which would contain an antiHermitian part if the field has an imaginary component. In [34] arguments are presented as to why the metric could be classical and complex-valued. For example, complex-valued effective metrics appear in modified gravity theories, when chiral deformations of general relativity are allowed [35]. Following this idea, we will explore the consequences of assuming a complex nonwhite classical noise coupled to the local mass density.

The paper is organized as follows. In Sec. II we derive, to the first meaningful perturbative order, the general collapse equation for the wave function, as well as the associated master equation in the case of $N$ complex-valued colored random noises $h_{i}(t)$, each coupled to an operator $\hat{A}_{i}$. The literature so far has considered only the case of real valued colored noises [11]. In Sec. III we show the collapse mechanism. In Sec. IV we consider specifically a noise field $w(\mathbf{x}, t)$ coupled to the local mass density $\hat{m}(\mathbf{x})$ and discuss the amplification mechanism, one of the crucial properties of any collapse model. In Sec. V we analyze the bounds on the spectrum of the noise, which are set by current experiments. We conclude the paper with a discussion of the results (Sec. VI).

\section{MASTER AND COLLAPSE EQUATIONS}

We have seen how the idea of a complex gravitational stochastic background inducing the collapse of the wave function leads to a collapse model where the noise is complex valued and, in general, colored. Since this has not been discussed in the literature so far, in this section we derive the appropriate collapse equation and the master equation, following the same strategy as in Appendix A for a real valued white noise. The starting point is the following generalized Schrödinger equation:

$$
i \hbar \partial_{t}\left|\phi_{t}\right\rangle=\left[\hat{H}_{0}+\xi \sum_{i=1}^{N} \hat{A}_{i} h_{i}(t)+\hat{O}\right]\left|\phi_{t}\right\rangle,
$$

where $\hat{A}_{i}$ are arbitrary self-adjoint operators and $h_{i}(t)$ are $N$ complex Gaussian noises, with zero average and correlation function

$$
\begin{aligned}
\mathbb{E}_{\mathbb{Q}}\left[h_{i}^{*}(t) h_{j}(\tau)\right] & =D_{i j}(t, \tau), \\
\mathbb{E}_{\mathbb{Q}}\left[h_{i}(t) h_{j}(\tau)\right] & =S_{i j}(t, \tau) .
\end{aligned}
$$

$D_{i j}(t, \tau)$ and $S_{i j}(t, \tau)$ are complex functions with magnitudes of order 1 , and $\hat{O}$ is an operator yet to be defined. The parameter $\xi$ sets the strength of the noise, which is assumed to be small. Following the scheme outlined in the introduction, we will determine $\hat{O}$ by the requirement of nonfaster-than-light signaling.

Since the norm of $\left|\phi_{t}\right\rangle$ is not conserved, we consider the normalized state $\left|\psi_{t}\right\rangle=\left|\phi_{t}\right\rangle / \| \phi_{t}||$, which solves the equation

$$
i \hbar \partial_{t}\left|\psi_{t}\right\rangle=\left(\hat{H}_{t}-\frac{1}{2}\left\langle\hat{H}_{t}-\hat{H}_{t}^{\dagger}\right\rangle_{t}\right)\left|\psi_{t}\right\rangle
$$

with

$$
\hat{H}_{t}=\hat{H}_{0}+\xi \sum_{i=1}^{N} \hat{A}_{i} h_{i}(t)+\hat{O} .
$$

As expected, the normalized vector evolves according to nonlinear stochastic dynamics. The stochastic ensemble of pure states $\rho_{t}^{h}=\left|\psi_{t}\right\rangle\left\langle\psi_{t}\right|$ obeys the following dynamics:

$$
\begin{aligned}
i \hbar \partial_{t} \rho_{t}^{h}= & {\left[\hat{H}_{0}+\xi \sum_{i=1}^{N}\left(\hat{A}_{i} h_{i}(t)-i\left\langle A_{i}\right\rangle_{t} h_{i}^{\mathrm{I}}(t)\right.\right.} \\
& \left.+\hat{O}-\frac{1}{2}\left\langle\hat{O}-\hat{O}^{\dagger}\right\rangle_{t}\right] \rho_{t}^{h}-\text { H.c. }
\end{aligned}
$$

Taking the expectation value to compute the dynamics for the density matrix $\rho_{t}=\mathbb{E}\left[\rho_{t}^{h}\right]$, one obtains, in general, a nonlinear evolution for the ensemble, which implies the possibility of faster-than-light signaling [21]. This can be avoided with a proper choice of the operator $\hat{O}$. Contrary to the white noise case, identifying the correct form of $\hat{O}$ is very difficult (in general, impossible) since the dependence of the right-hand side of the above equation on the noise $h$ is highly nontrivial. This means that one is not able to compute the stochastic average, and without such knowledge $\hat{O}$ cannot be determined. One way to circumvent the problem is to proceed perturbatively [36]. We Taylor-expand $\rho_{t}^{h}$ in terms of $\xi$,

$$
\rho_{t}^{h}=\rho_{0, t}^{h}+\xi \rho_{1, t}^{h}+\xi^{2} \rho_{2, t}^{h}+\mathcal{O}\left(\xi^{3}\right),
$$

where, for $t=0$, all terms except the first one are zero. We also expand $\hat{O}$ in powers of $\xi$ [37],

$$
\hat{O}=\xi \hat{O}_{1}+\xi^{2} \hat{O}_{2}+\mathcal{O}\left(\xi^{3}\right) .
$$

Exploiting the perturbative series above, one can find a closed equation for the average density $\mathbb{E}\left[\rho_{t}^{h}\right]$ and obtain the explicit expression (C5) for each term of the series (10), such that the average dynamic does not produce faster-than-light signaling (see Appendix C for the detailed calculation). This fixes the dynamical equation for the averaged density matrix to be, up to the second order in $\xi^{2}$, 
$\partial_{t} \rho_{t}=-\frac{i}{\hbar}\left[\hat{H}_{0}, \rho_{t}\right]-\frac{\xi^{2}}{\hbar^{2}}\left[\sum_{i, j=1}^{N} \int_{0}^{t} d \tau D_{i j}^{\mathrm{R}}(t, \tau)\left[\hat{A}_{i},\left[\hat{A}_{j}(\tau-t), \rho_{t}\right]\right]+i \sum_{i, j=1}^{N} \int_{0}^{t} d \tau D_{i j}^{\mathrm{I}}(t, \tau)\left[\hat{A}_{i},\left\{\hat{A}_{j}(\tau-t), \rho_{t}\right\}\right]\right]+\mathcal{O}\left(\xi^{3}\right)$,

where the superscript R/I stands for the real/imaginary part [38]. Exploiting then Eq. (C5) in Eq. (6), the collapse equation for the wave function turns out to be, up to second order in $\xi^{2}$,

$$
\begin{aligned}
i \hbar \partial_{t}\left|\psi_{t}\right\rangle= & {\left[\hat{H}_{0}+\xi\left(\sum_{i=1}^{N}\left(\hat{A}_{i} h_{i}(t)-i\left\langle A_{i}\right\rangle_{t} h_{i}^{\mathrm{I}}(t)\right)+\frac{i \xi^{2}}{\hbar} \sum_{i, j=1}^{N} \int_{0}^{t} d \tau\left(S_{i j}(t, \tau)-D_{i j}(t, \tau)\right) \hat{A}_{i}\left(\hat{A}_{j}(t-\tau)-\left\langle A_{j}(t-\tau)\right\rangle_{t}\right)\right.\right.} \\
& -\frac{i \xi^{2}}{\hbar} \sum_{i, j=1}^{N} \int_{0}^{t} d \tau\left(S_{i j}(t, \tau)-D_{i j}^{*}(t, \tau)\right)\left\langle A_{i}\right\rangle_{t} \hat{A}_{j}(\tau-t)-\frac{i \xi^{2}}{2 \hbar} \sum_{i, j=1}^{N} \int d \tau\left(S_{i j}(t, \tau)-D_{i j}(t, \tau)\right)\left(\left\langle A_{i} A_{j}(\tau-t)\right\rangle_{t}\right. \\
& \left.\left.-2\left\langle A_{i}\right\rangle_{t}\left\langle A_{j}(\tau-t)\right\rangle_{t}\right)-\frac{i \xi^{2}}{2 \hbar} \sum_{i, j=1}^{N} \int d \tau\left(S_{i j}^{*}(t, \tau)-D_{i j}^{*}(t, \tau)\right)\left(\left\langle A_{j}(\tau-t) A_{i}\right\rangle_{t}-2\left\langle A_{i}\right\rangle_{t}\left\langle A_{j}(\tau-t)\right\rangle_{t}\right)\right]\left|\psi_{t}\right\rangle .
\end{aligned}
$$

It is interesting to write down the Markovian limit, which is obtained by imposing $D_{i j}(t, s)=\delta(t-s) \tilde{D}_{i j}(t)$ and $S_{i j}(t, s)=\delta(t-s) \tilde{S}_{i j}(t)$; one ends up with the following stochastic Schrödinger equation in the Stratonovich form:

$$
\begin{aligned}
i \hbar \partial_{t}\left|\psi_{t}\right\rangle= & {\left[\hat{H}_{0}+\xi\left(\sum_{i=1}^{N}\left(\hat{A}_{i} h_{i}(t)-i\left\langle A_{i}\right\rangle_{t} h_{i}^{\mathrm{I}}(t)\right)+\frac{i \xi^{2}}{\hbar} \sum_{i, j=1}^{n}\left(\tilde{S}_{i j}(t)-\tilde{D}_{i j}(t)\right)\left[\left(\hat{A}_{i}-\left\langle A_{i}\right\rangle_{t}\right)\left(\hat{A}_{j}-\left\langle A_{j}\right\rangle_{t}\right)+\frac{1}{2}\left(\left\langle A_{i} A_{j}\right\rangle_{t}\right.\right.\right.\right.} \\
& \left.\left.\left.+\left\langle A_{j} A_{i}\right\rangle_{t}-2\langle A\rangle_{i}\langle A\rangle_{j}\right)\right]-\frac{i \xi^{2}}{\hbar} \sum_{i, j=1}^{N}\left(\tilde{S}_{i j}^{\mathrm{I}}(t)-\tilde{D}_{i j}^{\mathrm{I}}(t)\right)\left(\left\langle A_{i} A_{j}\right\rangle_{t}-2\left\langle A_{j} A_{i}\right\rangle_{t}\right)+\frac{i 2 \xi}{\hbar} \sum_{i, j=1}^{n} \tilde{D}_{i j}^{\mathrm{I}}(t)\left\langle A_{i}\right\rangle_{t} \hat{A}_{j}\right]\left|\psi_{t}\right\rangle .
\end{aligned}
$$

This equation is a generalization of Eq. (7.43) in [10]. The first two lines correspond to Eq. (7.43), with the replacement $\gamma \rightarrow \tilde{S}_{i j}(t)-\tilde{D}_{i j}(t)$, taking also into account that in our case the operators $A_{i}$ are not assumed to commute; the third line is associated with the complex part of the noise, while in [10] the noise was assumed to be real.

Equations (11) and (12) are the main result of this section, and will be used in the rest of the work.

In the next sections we will discuss the main consequences of Eqs. (11) and (12): the collapse of the wave function, the presence, under suitable conditions, of an amplification mechanism, and some experimental predictions.

\section{COLLAPSE OF THE WAVE FUNCTION}

We now establish under which conditions the dynamics given by Eq. (12), when $H_{0}=0$, induce the collapse of the state vector $|\psi\rangle_{t}$ into one of the eigenstates of $\hat{A}_{i}$, assuming that these operators commute with each other and therefore have a common set of eigenstates. We will follow the procedure outlined in Sec. II a of [36]. We neglect the standard evolution since we are focusing only on the collapse process. This approximation, in general not true, is good for macroscopic objects. In fact, given the amplification mechanism, which we will describe in the next section, the effect of the collapse increases with the mass of the system, becoming dominant with respect to the standard evolution for large objects.
We consider the stochastic average of the variance $V_{A}(t)=\left\langle\hat{A}^{2}\right\rangle_{t}-\langle\hat{A}\rangle_{t}^{2}$ of an operator $\hat{A}$ which commutes with all $\hat{A}_{i}$. One may prove that, for any $n$,

$$
\mathbb{E}\left[\left\langle\hat{A}^{n}\right\rangle_{t}\right]=\operatorname{Tr}\left[\rho_{t} \hat{A}^{n}\right]=\operatorname{Tr}\left[\rho_{0} \hat{A}^{n}\right]=\mathbb{E}\left[\left\langle\hat{A}^{n}\right\rangle_{0}\right] .
$$

Then, exploiting the perturbative series in Eq. (C2) and performing the stochastic average, one can obtain

$$
\begin{aligned}
\mathbb{E}\left[\langle A\rangle_{t}^{2}\right]= & \mathbb{E}\left[\langle A\rangle_{0}\right] \\
& -\frac{2 \xi^{2}}{\hbar^{2}} \sum_{i, j=1}^{N} \int_{0}^{t} d \tau \int_{0}^{\tau} d s\left(S_{i j}^{\mathrm{R}}(\tau, s)-D_{i j}^{\mathrm{R}}(\tau, s)\right) \\
& \times\left\langle\langle A \rangle _ { 0 } ( A _ { i } - \langle A _ { i } \rangle _ { 0 } \rangle _ { 0 } \left\langle\langle A\rangle_{0}\left(A_{j}-\left\langle A_{j}\right\rangle_{0}\right\rangle_{0}\right.\right. \\
& +\mathcal{O}\left(\xi^{3}\right) .
\end{aligned}
$$

Given the above result, one can now compute the stochastic average of the variance $V_{A}(t)$, arriving at

$$
\begin{aligned}
\mathbb{E}\left[V_{A}(t)\right]= & V_{A}(0)-\frac{2 \xi^{2}}{\hbar^{2}} \sum_{i, j=1}^{N} \int_{0}^{t} d \tau F_{i j}(\tau) \\
& \left.\times\left(\left\langle A A_{i}\right\rangle_{0}-\langle A\rangle_{0}\left\langle A_{i}\right\rangle_{0}\right)\left(\left\langle A A_{j}\right)\right\rangle_{0}-\langle A\rangle_{0}\left\langle A_{j}\right\rangle_{0}\right) \\
& +\mathcal{O}\left(\xi^{3}\right),
\end{aligned}
$$

where 


$$
F_{i j}(\tau)=\int_{0}^{\tau} d \mathcal{S}\left(D_{i j}^{\mathrm{R}}(\tau, s)-S_{i j}^{\mathrm{R}}(\tau, s)\right) .
$$

According to [39] the positivity of $F(\mathbf{x}, \mathbf{y}, \tau)$ in the limit $t \rightarrow \infty$ is a sufficient condition to guarantee the reduction properties of Eq. (12). In fact, whenever $F$ is non-negative, Eq. (16) implies that, for large times the covariance $\left(\left\langle A A_{i}\right\rangle_{\tau}-\langle A\rangle_{\tau}\left\langle A_{i}\right\rangle_{\tau}\right)$ converges to 0 for any realization of the noise, with the only possible exception of a subset of measure 0 . In particular, when $\hat{A}$ is equal to $A_{i}$, we have

$$
\lim _{t \rightarrow \infty}\left\langle A_{i} A_{i}\right\rangle_{t}-\left\langle A_{i}\right\rangle_{t}\left\langle A_{i}\right\rangle_{t}=\lim _{t \rightarrow \infty} V_{A_{i}}(t)=0 .
$$

This means that any initial state converges asymptotically, with probability 1 , to one of the eigenstates of the operator $\hat{A_{i}}$.

A related question is how fast the wave function collapses. The decoherence rate of the associated master Eq. (11) provides a good measure. If we set $\hat{H}_{0}=0$, we immediately obtain the decoherence rate in the basis of the common eigenstates of the operators $\hat{A}_{i}$,

$$
\begin{aligned}
\rho_{t}(\alpha, \beta)= & \exp \left(-\frac{\xi^{2}}{\hbar^{2}} \sum_{i, j=1}^{N} \int_{0}^{t} d \tau \int_{0}^{\tau} d s D_{i j}^{\mathrm{R}}(\tau, s)\left(\alpha_{i} \alpha_{j}-\alpha_{i} \beta_{j}-\alpha_{j} \beta_{i}+\beta_{j} \beta_{i}\right)\right. \\
& \left.+i D_{i j}^{\mathrm{I}}(\tau, s)\left(\alpha_{i} \alpha_{j}+\alpha_{i} \beta_{j}+\alpha_{j} \beta_{i}+\beta_{j} \beta_{i}\right)\right) \rho_{0}(\alpha, \beta) \\
= & \exp \left(-\frac{\xi^{2}}{\hbar^{2}} \sum_{i, j=1}^{N} \int_{0}^{t} d \tau \int_{0}^{\tau} d s D_{i j}(\tau, s)\left(\alpha_{i} \alpha_{j}-\beta_{i} \beta_{j}\right)-D_{i, j}^{*}(\tau, s)\left(\alpha_{i} \beta_{j}+\alpha_{j} \beta_{i}\right)\right) \rho_{0}(\alpha, \beta),
\end{aligned}
$$

where $\rho_{t}(\alpha, \beta)=\left\langle\alpha\left|\rho_{t}\right| \beta\right\rangle$ and $|\alpha\rangle(|\beta\rangle)$ is one element of the basis, i.e., $\hat{A}_{i}|\alpha\rangle=\alpha_{i}|\alpha\rangle$.

It is worth studying the case where there is only one collapse operator and the correlation is real and delta correlated in time, i.e.,

$$
D(\tau, s)=\tau_{0} \delta(\tau-s),
$$

with $\tau_{0}$ a real parameter with the dimensions of a time. Then Eq. (19) reduces to

$$
\rho_{t}(\alpha, \beta)=e^{-\frac{\xi^{2} \tau_{0} t}{\hbar^{2}}(\alpha-\beta)^{2}} \rho_{0}(\alpha, \beta),
$$

where the decoherence rate is constant in time and is determined by $\tau_{0} \xi^{2}$.

\section{MASTER EQUATION FOR THE CENTER OF MASS AND THE AMPLIFICATION MECHANISM}

After the collapse of the wave function, the next fundamental requirement for a good collapse model is the amplification mechanism: the center of mass wave function of a composite system should collapse with a rate which increases with the size of the system. This is necessary in order for the equation to preserve the quantum properties of microscopic systems and, at the same time, to guarantee the classical properties of macroscopic objects.

Instead of considering the problem in full generality as done in the previous two sections, we focus our analysis to the case of interest here: the collapse noise coupled to the mass density operator $\hat{m}(\mathbf{x})$. In this case Eq. (11) takes the form

$$
\begin{aligned}
\partial_{t} \rho_{t}= & -\frac{i}{\hbar}\left[\hat{H}_{0}, \rho_{t}\right]-\frac{\xi^{2} c^{4}}{\hbar^{2}} \int d \mathbf{x} \int d \mathbf{y} \int_{0}^{t} d \tau D^{\mathrm{R}}(\mathbf{x}-\mathbf{y}, t-\tau) \\
& \times\left[\hat{m}(\mathbf{x}),\left[\hat{m}(\mathbf{y}, \tau-t), \rho_{t}\right]\right] \\
& -\frac{i \xi^{2} c^{4}}{\hbar^{2}} \int d \mathbf{x} \int d \mathbf{y} \int_{0}^{t} d \tau D^{\mathrm{I}}(\mathbf{x}-\mathbf{y}, t-\tau) \\
& \times\left[\hat{m}(\mathbf{x}),\left\{\hat{m}(\mathbf{y}, \tau-t), \rho_{t}\right\}\right],
\end{aligned}
$$

where $D^{\mathrm{R}}$ and $D^{\mathrm{I}}$ are the real and the imaginary parts of the correlation function of the noise field [40] $D(\mathbf{x}, \mathbf{y} ; t, \tau)=\mathbb{E}\left[h^{*}(\mathbf{x}, t) h(\mathbf{y}, \tau)\right]$. In writing the above equation, we assumed that the noise is statistically homogeneous over space and time: $D^{\mathrm{R}, \mathrm{I}}(\mathbf{x}, \mathbf{y}, t, \tau)=D^{\mathrm{R}, \mathrm{I}}(\mathbf{x}-\mathbf{y}, t-\tau)$. We consider a system of $N$ pointlike particles. The mass density function is

$$
\begin{aligned}
\hat{m}(\mathbf{x}) & =\sum_{i=1}^{N} m_{i} \delta\left(\mathbf{x}-\hat{\mathbf{x}}_{i}\right) \\
& =\sum_{i=1}^{N} \frac{m_{i}}{(2 \pi \hbar)^{3}} \int d \mathbf{Q} e^{\frac{i}{h} \mathbf{Q} \cdot\left(\mathbf{x}-\hat{\mathbf{x}}_{i}\right)} .
\end{aligned}
$$

Substituting Eq. (23) into Eq. (22) and performing the integration over $\mathbf{x}$ and $\mathbf{y}$, we arrive at the expression

$$
\begin{aligned}
\partial_{t} \rho_{t}= & -\frac{i}{\hbar}\left[\hat{H}_{0}, \rho_{t}\right]-\frac{\xi^{2} c^{4}}{\hbar^{2}} \sum_{i, j=1}^{N} \frac{m_{i} m_{j}}{(2 \pi \hbar)^{3}} \int_{0}^{t} d \tau \int d \mathbf{Q} \tilde{D}^{\mathrm{R}}(\mathbf{Q}, t-\tau) \\
& \times\left[e^{-\frac{i}{\hbar} \mathbf{Q} \cdot \hat{\mathbf{x}}_{i}},\left[e^{\frac{i}{\hbar} \hat{H}_{0}(\tau-t)} e^{\frac{i}{\hbar} \mathbf{Q} \cdot \hat{\mathbf{x}}_{j}} e^{-\frac{i}{\hbar} \hat{H}_{0}(\tau-t)}, \rho_{t}\right]\right] \\
& -\frac{i \xi^{2} c^{4}}{\hbar^{2}} \sum_{i, j=1}^{N} \frac{m_{i} m_{j}}{(2 \pi \hbar)^{3}} \int_{0}^{t} d \tau \int d \mathbf{Q} \tilde{D}^{\mathrm{I}}(\mathbf{Q}, t-\tau) \\
& \times\left[e^{-\frac{i}{\hbar} \mathbf{Q} \cdot \hat{\mathbf{x}}_{i}},\left\{e^{\frac{i}{\hbar} \hat{H}_{0}(\tau-t)} e^{\frac{i}{\hbar} \mathbf{Q} \cdot \hat{\mathbf{x}}_{j}} e^{-\frac{i}{\hbar} \hat{H}_{0}(\tau-t)}, \rho_{t}\right\}\right]
\end{aligned}
$$


where we defined

$$
\tilde{D}^{\beta}(\mathbf{Q}, t-\tau):=\int d \mathbf{r} D^{\beta}(\mathbf{r}, t-\tau) e^{\frac{i}{\hbar} \mathbf{Q} \cdot \mathbf{r}}
$$

with $\beta=\mathrm{R}, \mathrm{I}$.

We are interested in describing the dynamics of the center of mass of the composite system. In particular, we have in mind the case of a rigid body. We introduce the center of mass coordinates

$$
\hat{\mathbf{X}}=\sum_{i=1}^{N} \frac{m_{i}}{M} \hat{\mathbf{x}}_{i}, \quad \hat{\mathbf{P}}=\sum_{i=1}^{N} \hat{\mathbf{q}_{i}},
$$

and the relative coordinates

$$
\begin{cases}\hat{\boldsymbol{r}}_{i}=\hat{\mathbf{x}_{i}}-\hat{\mathbf{X}} & i \in(1, \ldots, N-1), \\ \hat{\boldsymbol{r}}_{N}=-\sum_{i=1}^{N-1} \frac{m_{i}}{m_{N}} \hat{\boldsymbol{r}}_{i}, & \\ \hat{\mathbf{p}_{i}}=\hat{\mathbf{q}}_{i}-\frac{m_{i}}{M} \hat{\mathbf{P}} & i \in(1, \ldots, N-1), \\ \hat{\mathbf{p}}_{N}=-\sum_{i=1}^{N-1} \hat{\mathbf{p}_{i},} & \end{cases}
$$

where $M=\sum_{i=1}^{N} m_{i}$ is the total mass of the system. The operators $\hat{\boldsymbol{r}}_{N}$ and $\hat{\mathbf{p}}_{N}$ are not independent (they are defined in terms of the other relative positions and momenta) but it is convenient to keep them to make the notation simpler. These new variables obey the following commutation relations:

$$
\begin{aligned}
& {[\hat{\mathbf{X}}, \hat{\mathbf{P}}]=i \hbar \quad\left[\hat{\boldsymbol{r}}_{i}, \hat{\mathbf{p}}_{j}\right]=i \hbar\left(\delta_{i j}-\frac{m_{i}}{M}\right)} \\
& {\left[\hat{\mathbf{X}}, \hat{\boldsymbol{r}}_{i}\right]=\left[\hat{\mathbf{X}}, \hat{\mathbf{p}}_{i}\right]=\left[\hat{\boldsymbol{r}}_{i}, \hat{\boldsymbol{r}}_{j}\right]=\left[\hat{\boldsymbol{r}_{i}}, \hat{\mathbf{P}}\right]=0}
\end{aligned}
$$

for $i, j \in(1, \ldots, N-1)$. We introduce the center of mass density matrix as

$$
\rho_{t}^{\mathrm{CM}}:=\operatorname{Tr}_{\mathrm{REL}}\left(\rho_{t}\right),
$$

where $\operatorname{Tr}_{\text {REL }}(\cdot)$ denotes the partial trace over the relative coordinates. We study the effect of the partial trace on the operators of Eq. (24). Assuming that $\hat{H}_{0}=\hat{H}_{0}^{\mathrm{CM}}+\hat{H}_{0}^{\mathrm{REL}}$, the term in the first line simplifies as

$$
\operatorname{Tr}_{\mathrm{REL}}\left(\left[\hat{H}_{0}^{\mathrm{CM}}+\hat{H}_{0}^{\mathrm{REL}}, \rho_{t}\right]\right)=\left[\hat{H}_{0}^{\mathrm{CM}}, \rho_{t}^{\mathrm{CM}}\right] .
$$

The double commutator in the third line can be expanded as the sum of four terms,

$$
\begin{aligned}
\operatorname{Tr}_{\mathrm{REL}} & \left(\left[e^{-\frac{i}{\hbar} \mathbf{Q} \cdot \hat{\mathbf{x}}_{i}},\left[e^{\frac{i}{\hbar} \hat{H}_{0}(\tau-t)} e^{\frac{i}{\hbar} \mathbf{Q} \cdot \hat{\mathbf{x}}_{j}} e^{-\frac{i}{\hbar} \hat{H}_{0}(\tau-t)}, \rho_{t}\right]\right]\right) \\
= & \operatorname{Tr}_{\mathrm{REL}}\left(e^{-\frac{i}{\hbar} \mathbf{Q} \cdot \hat{\mathbf{x}}_{i}} e^{\frac{i}{\hbar} \hat{H}_{0}(\tau-t)} e^{\frac{i}{\hbar} \mathbf{Q} \cdot \hat{\mathbf{x}}_{j}} e^{-\frac{i}{\hbar} \hat{H}_{0}(\tau-t)} \rho_{t}\right) \\
& -\operatorname{Tr}_{\operatorname{REL}}\left(e^{\frac{i}{\hbar} \hat{H}_{0}(\tau-t)} e^{\frac{i}{\hbar} \mathbf{Q} \cdot \hat{\mathbf{x}}_{j}} e^{-\frac{i}{\hbar} \hat{H}_{0}(\tau-t)} \rho_{t} e^{-\frac{i}{\hbar} \mathbf{Q} \cdot \hat{\mathbf{x}}_{i}}\right) \\
& -\operatorname{Tr}_{\operatorname{REL}}\left(e^{-\frac{i}{\hbar} \mathbf{Q} \cdot \hat{\mathbf{x}}_{i}} \rho_{t} e^{\frac{i}{\hbar} \hat{H}_{0}(\tau-t)} e^{\frac{i}{\hbar} \mathbf{Q} \cdot \hat{\mathbf{x}}_{j}} e^{-\frac{i}{\hbar} \hat{H}_{0}(\tau-t)}\right) \\
& +\operatorname{Tr}_{\operatorname{REL}}\left(\rho_{t} e^{\frac{i}{\hbar} \hat{H}_{0}(\tau-t)} e^{\frac{i}{h} \mathbf{Q} \cdot \hat{\mathbf{x}}_{j}} e^{-\frac{i}{\hbar} \hat{H}_{0}(\tau-t)} e^{-\frac{i}{\hbar} \mathbf{Q} \cdot \hat{\mathbf{x}}_{i}}\right) .
\end{aligned}
$$

We consider the first term on the right-hand side, as the calculations for the remaining terms are similar. Exploiting the commutativity of the relative and center of mass degree of freedoms, we rewrite the exponential operators in Eq. (29) as $e^{-\frac{i}{\hbar} \mathbf{Q} \cdot \hat{\mathbf{x}}_{i}}=e^{-\frac{i}{\hbar} \mathbf{Q} \cdot \hat{\boldsymbol{X}}} e^{-\frac{i}{\hbar} \mathbf{Q} \cdot \hat{r}_{i}}, \quad e^{\frac{i}{\hbar} \hat{H}_{0}(\tau-t)}=e^{\frac{i}{\hbar} \hat{H}_{0}^{\mathrm{CM}}(\tau-t)} e^{\frac{i}{\hbar} \hat{H}_{0}^{\mathrm{REL}}(\tau-t)}$,

so that

$$
\begin{aligned}
\operatorname{Tr}_{\mathrm{REL}}\left(e^{-\frac{i}{\hbar} \mathbf{Q} \cdot \hat{\mathbf{x}}_{i}} e^{\frac{i}{\hbar} \hat{H}_{0}(\tau-t)} e^{\frac{i}{\hbar} \mathbf{Q} \cdot \hat{\mathbf{x}}_{j}} e^{-\frac{i}{\hbar} \hat{H}_{0}(\tau-t)} \rho_{t}\right) \\
=\operatorname{Tr}_{\mathrm{REL}}\left(\left[e^{-\frac{i}{\hbar} \mathbf{Q} \cdot \hat{r}_{i}} e^{\frac{i}{\hbar} \hat{H}_{0}^{\mathrm{REL}}(\tau-t)} e^{\frac{i}{\hbar} \mathbf{Q} \cdot \hat{r}_{j}} e^{-\frac{i}{\hbar} \hat{H}_{0}^{\mathrm{REL}}(\tau-t)}\right]\right. \\
\left.\quad \times\left[e^{-\frac{i}{\hbar} \mathbf{Q} \cdot \hat{\boldsymbol{X}}} e^{\frac{i}{\hbar} \hat{H}_{0}^{\mathrm{CM}}(\tau-t)} e^{\frac{i}{\hbar} \mathbf{Q} \cdot \hat{\boldsymbol{x}}} e^{-\frac{i}{\hbar} \hat{H}_{0}^{\mathrm{CM}}(\tau-t)}\right] \rho_{t}\right) .
\end{aligned}
$$

We assume the motion of the relative coordinates to be a small fluctuation around the equilibrium positions $\boldsymbol{r}_{i 0}$ within the solid (e.g., in a crystalline structure), i.e., $\hat{\boldsymbol{r}}_{i}(t)=$ $\boldsymbol{r}_{i 0}+\Delta \hat{\boldsymbol{r}}_{i}(t)$, where the fluctuations $\Delta \hat{\boldsymbol{r}}_{i}(t)$ are negligible with respect to the spatial correlation length of the noise within the time $t-\tau$. Under this approximation, the square bracket in the second line of Eq. (31) becomes $e^{-\frac{i}{\hbar} \mathbf{Q} \cdot\left(\boldsymbol{r}_{i 0}-\boldsymbol{r}_{j 0}\right)}$, and we obtain

$$
\begin{aligned}
& \operatorname{Tr}_{\mathrm{REL}}\left(e^{-\frac{i}{\hbar} \mathbf{Q} \cdot \hat{\mathbf{x}}_{i}} e^{\frac{i}{\hbar} \hat{H}_{0}(\tau-t)} e^{\frac{i}{\hbar} \mathbf{Q} \cdot \hat{\mathbf{x}}_{j}} e^{-\frac{i}{\hbar} \hat{H}_{0}(\tau-t)} \rho_{t}\right) \\
& \quad \simeq e^{-\frac{i}{\hbar} \mathbf{Q} \cdot\left(\boldsymbol{r}_{i 0}-\boldsymbol{r}_{j 0}\right)} e^{-\frac{i}{\hbar} \mathbf{Q} \cdot \hat{\boldsymbol{x}}} e^{\frac{i}{\hbar} \hat{H}_{0}^{\mathrm{CM}}(\tau-t)} e^{\frac{i}{\hbar} \mathbf{Q} \cdot \hat{\boldsymbol{x}}} e^{-\frac{i}{\hbar} \hat{H}_{0}^{\mathrm{CM}}(\tau-t)} \rho_{t}^{\mathrm{CM}},
\end{aligned}
$$

which depends on center of mass operators only. The other three terms on the right-hand side in Eq. (29) can be computed in the same way, and therefore we get the overall result

$$
\begin{aligned}
& \operatorname{Tr}_{\mathrm{REL}}\left(\left[e^{-\frac{i}{\hbar} \mathbf{Q} \cdot \hat{\mathbf{x}}_{i}},\left[e^{\frac{i}{\hbar} \hat{H}_{0}(\tau-t)} e^{\frac{i}{\hbar} \mathbf{Q} \cdot \hat{\mathbf{x}}_{j}} e^{-\frac{i}{\hbar} \hat{H}_{0}(\tau-t)}, \rho_{t}\right]\right]\right) \\
& \quad=e^{-\frac{i}{\hbar} \mathbf{Q} \cdot\left(\boldsymbol{r}_{i 0}-\boldsymbol{r}_{j 0}\right)}\left[e^{-\frac{i}{\hbar} \mathbf{Q} \cdot \hat{\boldsymbol{X}}},\left[e^{\frac{i}{\hbar} \hat{H}_{0}^{\mathrm{CM}}(\tau-t)} e^{\frac{i}{\hbar} \mathbf{Q} \cdot \hat{\boldsymbol{X}}} e^{-\frac{i}{\hbar} \hat{H}_{0}^{\mathrm{CM}}(\tau-t)}, \rho_{t}^{\mathrm{CM}}\right]\right] .
\end{aligned}
$$

Similarly, for the operators in the fifth line of Eq. (24) we obtain

$$
\begin{aligned}
& \operatorname{Tr}_{\mathrm{REL}}\left(\left[e^{-\frac{i}{\hbar} \mathbf{Q} \cdot \hat{\mathbf{x}}_{i}},\left\{e^{\frac{i}{\hbar} \hat{H}_{0}(\tau-t)} e^{\frac{i}{\hbar} \mathbf{Q} \cdot \hat{\mathbf{x}}_{j}} e^{-\frac{i}{\hbar} \hat{H}_{0}(\tau-t)}, \rho_{t}\right\}\right]\right) \\
& \quad=e^{-\frac{i}{\hbar} \mathbf{Q} \cdot\left(\boldsymbol{r}_{i 0}-\boldsymbol{r}_{j 0}\right)}\left[e^{-\frac{i}{\hbar} \mathbf{Q} \cdot \hat{\boldsymbol{X}}},\left\{e^{\frac{i}{\hbar} \hat{H}_{0}^{\mathrm{CM}}(\tau-t)} e^{\frac{i}{\hbar} \mathbf{Q} \cdot \hat{\boldsymbol{X}}} e^{-\frac{i}{\hbar} \hat{H}_{0}^{\mathrm{CM}}(\tau-t)}, \rho_{t}^{\mathrm{CM}}\right\}\right] .
\end{aligned}
$$

Combining the previous results, we arrive at the following master equation for the center of mass

$$
\begin{aligned}
\partial_{t} \rho_{t}^{\mathrm{CM}}= & -\frac{i}{\hbar}\left[\hat{H}_{0}^{\mathrm{CM}}, \rho_{t}^{\mathrm{CM}}\right] \\
& -\frac{\xi^{2} c^{4}}{\hbar^{2}} \frac{1}{(2 \pi \hbar)^{3}} \int_{0}^{t} d \tau \int d \mathbf{Q} \tilde{D}^{\mathrm{R}}(\mathbf{Q}, t-\tau) A(\mathbf{Q}) \\
& \times\left[e^{-\frac{i}{\hbar} \mathbf{Q} \cdot \hat{\boldsymbol{X}}},\left[e^{\frac{i}{\hbar} \hat{H}_{0}^{\mathrm{CM}}(\tau-t)} e^{\frac{i}{\hbar} \mathbf{Q} \cdot \hat{\boldsymbol{X}}} e^{-\frac{i}{\hbar} \hat{H}_{0}^{\mathrm{CM}}(\tau-t)}, \rho_{t}^{\mathrm{CM}}\right]\right] \\
& -\frac{i \xi^{2} c^{4}}{\hbar^{2}} \frac{1}{(2 \pi \hbar)^{3}} \int_{0}^{t} d \tau \int d \mathbf{Q} \tilde{D}^{\mathrm{I}}(\mathbf{Q}, t-\tau) A(\mathbf{Q}) \\
& \times\left[e^{-\frac{i}{\hbar} \mathbf{Q} \cdot \hat{\boldsymbol{X}}},\left\{e^{\frac{i}{\hbar} \hat{H}_{0}^{\mathrm{CM}}(\tau-t)} e^{\frac{i}{\hbar} \mathbf{Q} \cdot \hat{\boldsymbol{X}}} e^{-\frac{i}{\hbar} \hat{H}_{0}^{\mathrm{CM}}(\tau-t)}, \rho_{t}^{\mathrm{CM}}\right\}\right],
\end{aligned}
$$

with 


$$
A(\mathbf{Q}):=\sum_{i, j=1}^{N} m_{i} m_{j} e^{-\frac{i}{\hbar} \mathbf{Q} \cdot\left(\boldsymbol{r}_{i 0}-\boldsymbol{r}_{j 0}\right)}=|\rho(\mathbf{Q} / \hbar)|^{2},
$$

where

$$
\rho(\mathbf{k}):=\int d \mathbf{x} \rho(\mathbf{x}) e^{-i \mathbf{k} \mathbf{x}}
$$

is the Fourier transform of the classical mass density distribution $\rho(\mathbf{x}):=\sum_{i=1}^{N} m_{i} \delta\left(\mathbf{x}-\boldsymbol{r}_{i}^{\mathrm{cl}}\right)$.

The master equation (32) for the center of mass wave function has the same structure as the single particle master equation, with the addition of the amplifying factor $A(\mathbf{Q})$, which keeps track of the fact that we are dealing with a composite object, not a pointlike particle.

Typically, the noise correlators $D^{\mathrm{R}}(\mathbf{r}, t-\tau)$ and $D^{\mathrm{I}}(\mathbf{r}, t-\tau)$ are expected to have spatial cutoffs (the noise correlation length), respectively $r_{C}^{\mathrm{R}}$ and $r_{C}^{\mathrm{I}}$. As for the case of the continuous spontaneous localization (CSL) model [9], it is interesting to study the behavior of the amplification factor in two limiting cases (for a more detailed proof of what follows, see [41]):

(1) When the particles are at distances smaller than the noise correlation lengths $r_{C}^{\mathrm{R}}, r_{C}^{\mathrm{I}}$, they contribute coherently, giving a factor $\propto\left(\sum_{i} m_{i}\right)^{2}$.

(2) When the particles are at distances larger than the noise correlation lengths $r_{C}^{\mathrm{R}}, r_{C}^{\mathrm{I}}$, they contribute incoherently, giving a factor $\propto \sum_{i} m_{i}^{2}$.

Because of these two properties, a reasonable estimate of the amplification factor in Eq. (33), is provided by Adler's formula [41,42],

$$
A^{\beta}=A^{\beta}\left(r_{C}^{\beta}\right)=N^{\beta}\left(n^{\beta} m_{0}\right)^{2} \quad \text { with } \quad \beta=\mathrm{R}, \mathrm{I},
$$

where $A^{\beta}$ refers to $A$ in the second line of Eq. (32) for $\beta=\mathrm{R}$ and to $A$ in the fourth line for $\beta=\mathrm{I} ; n^{\beta}$ is the number of nucleons of mass $m_{0}$ inside a sphere of radius $r_{C}^{\beta}$, while $N^{\beta}$ denotes the number of such spheres necessary for covering the entire object.

\section{EXPERIMENTAL BOUNDS ON THE GRAVITATIONAL NOISE SPECTRUM}

Discussing the experimental constraints on the noise correlator in its full generality is too difficult. We will limit the discussion to a restricted class of Gaussian correlation functions, in such a way that the collapse dynamics is controlled by only two parameters (for a class of correlation function that leads to a Hu-Paz-Zhang (HPZ) type master equation, see Appendix D).

Specifically, we consider the Markovian limit by imposing

$$
\tilde{D}^{\mathrm{R}}(\mathbf{Q}, s) \approx \tilde{D}^{\mathrm{R}}(\mathbf{Q}) \tau_{0} \delta(s),
$$

with $\left[\tau_{0}\right]=[T]$ [see Eq. (20)]. From the definition of $D_{i j}(t, \tau)$ in Eq. (5), using the definition of the Fourier transform and Eq. (36), it is straightforward to show that $\tilde{D}^{\mathrm{I}}(\mathbf{Q})=0$. In addition, to make contact with existing phenomenology for the CSL model [9], we assume that $\tilde{D}^{\mathrm{R}}(\mathbf{Q})$ has the following form:

$$
\tilde{D}^{\mathrm{R}}(\mathbf{Q})=r_{C}^{3} \exp \left(-r_{C}^{2} \mathbf{Q}^{2} / \hbar^{2}\right),
$$

where $\left[r_{C}\right]=[L]$. With these assumptions, after some algebra, Eq. (32) reduces to

$$
\begin{aligned}
\partial_{t} \rho_{t}^{\mathrm{CM}}= & -\frac{i}{\hbar}\left[\hat{H}_{0}^{\mathrm{CM}}, \rho_{t}^{\mathrm{CM}}\right]-\frac{\xi^{2} c^{4} r_{C}^{3} \tau_{0}}{(2 \pi \hbar)^{3} 2 \hbar^{2}} \\
& \times \int d \mathbf{Q} A(\mathbf{Q}) \exp \left(-r_{C}^{2} Q^{2} / \hbar^{2}\right) \\
& \times\left[e^{-\frac{i}{\hbar} \mathbf{Q} \cdot \hat{X}},\left[e^{\frac{i}{\hbar} \mathbf{Q} \cdot \hat{X}}, \rho_{t}^{\mathrm{CM}}\right]\right] .
\end{aligned}
$$

This equation should be compared with the CSL master equation [9]

$$
\begin{aligned}
\partial_{t} \rho_{t}^{\mathrm{CM}}= & -\frac{i}{\hbar}\left[\hat{H}_{0}^{\mathrm{CM}}, \rho_{t}^{\mathrm{CM}}\right]-\frac{\lambda\left(4 \pi r_{C}^{2}\right)^{3 / 2}}{(2 \pi \hbar)^{3}} \int d \mathbf{Q} \frac{A(\mathbf{Q})}{m_{0}^{2}} \\
& \times \exp \left(-r_{C}^{2} \mathbf{Q}^{2} / \hbar^{2}\right)\left[e^{-\frac{i}{\hbar} \mathbf{Q} \cdot \hat{\boldsymbol{X}}},\left[e^{\frac{i}{\hbar} \mathbf{Q} \cdot \hat{\boldsymbol{X}}}, \rho_{t}^{\mathrm{CM}}\right]\right] .
\end{aligned}
$$

In particular, Eq. (38) reduces to the CSL master equation given in Eq. (39) by setting

$$
\xi=\frac{4 \hbar \pi^{3 / 4}}{m_{0} c^{2}} \sqrt{\frac{\lambda}{\tau_{0}}} .
$$

To simplify the discussion, we assume the time cutoff to be related to the space cutoff via $\tau_{0}=r_{C} / c$. We can now set bounds on $\left(\xi, r_{C}\right)$ [or equivalently on $\left(\xi, \tau_{0}\right)$ ] by using the bounds already set for the CSL parameters $\left(\lambda, r_{C}\right)$. We have summarized the most recent bounds in Fig. 1.

The primary feature of any good collapse model is to suppress macroscopic linear superpositions. By choosing an appropriate macroscopicity or classicality scale, one can estimate the minimal strength the collapse should have. Specifically, the lower bound (Macro) in Fig. 1 is obtained by requiring that an object of size $0.01 \mathrm{~mm}$ is localized within $10 \mathrm{~ms}[41,46]$. This means that, more or less, the smallest object visible to the naked eye is localized within the perception time of a human observer. Needless to say, this bound can change by several orders of magnitude depending on the chosen criteria of classicality.

The coupling with the noise field not only suppresses macroscopic superposition but, as a side effect, also makes particles constantly jiggle, and this random motion can be tested by noninterferometric experiments [47-49]. Here we consider some of the most relevant such experiments, 


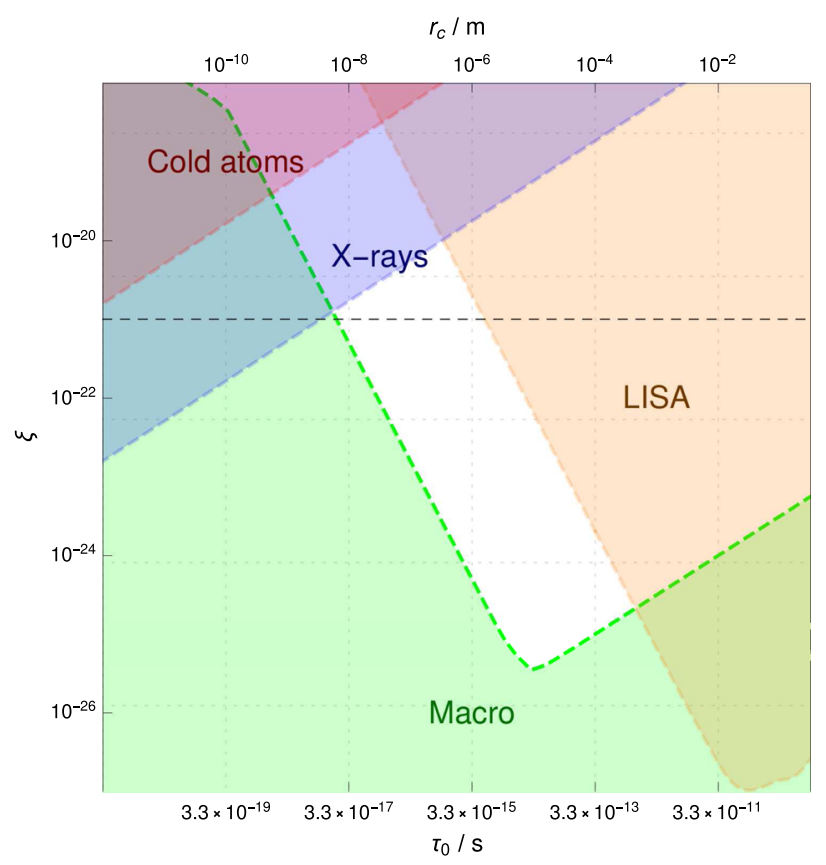

FIG. 1. $\left(\xi, r_{C}\right)$ or equivalently $\left(\xi, \tau_{0}\right)$ parameter diagram of the gravity induced collapse model in the Markovian regime given by Eq. (38). The white area is the allowed region. The other shaded regions are excluded: the orange-shaded region (LISA) from the data analysis of LISA Pathfinder [43], the blue-shaded region (X-rays) from data analysis of X-rays measurements [44], the purple-shaded region from the data analysis with cold atom experiments (Cold atoms) [45]. The green shaded region (Macro) is obtained by requiring that the collapse is strong enough to localize macroscopic objects $[41,46]$. As a reference, the horizontal dashed line is the magnitude of the real gravitational waves recently discovered by LIGO.

which set rather stringent bounds on the collapse parameters. The results are summarized in Fig. 1.

Consider first a charged particle: It is expected that the random jitters (accelerations) make it emit photons. The absence of this extra radiation, as it can be extracted by analyzing the spectrum of emission from Germanium measured over long times [44], can then be used to obtain very good bounds (X-rays).

Another interesting bound on the size of the Brownian motion induced by the collapse mechanism comes from accurate monitoring of the motion of relatively large masses, as is the case of the LISA pathfinder experiment [43]. These bounds have been obtained from the bounds on the parameters of the Markovian CSL model. However, for such a large object, we expect that the dynamics do not change significantly when we consider a colored noise: the relevant time scale of evolution is much longer than the considered values of the noise correlation time.

The last bound we consider is derived by studying the evolution of a gas of cold atoms. The collapse induced jiggling makes the gas expand more than what is predicted by standard quantum mechanics, and this difference becomes appreciable if the gas is initially at very low temperature. The absence of any appreciable difference [45] gives the bound denoted by "Cold atoms" in Fig. 1. Although this bound is less strong than the one obtained from the X-ray experiment, it is the only one which has been shown to also persist for a non-Markovian noise field.

We leave a more refined analysis of the other bounds, in the regime where non-Markovian are expected to become important, for future research.

We compare these results with the recent discovery of gravitational waves [50], observed in frequency range from 35 to $250 \mathrm{~Hz}$ and with a peak strain of $1.0 \times 10^{-21}$. Clearly, gravitational waves are real, while here the claim is that the collapse is caused by complex fluctuations of the metric. Also, gravitational waves typically have longer wavelengths, while here the relevant part of the spectrum is at high frequencies (Fig. 1). However, it is interesting to see that in order to have an efficient wave function collapse, the complex fluctuations need not be very strong. They can well be several orders of magnitude weaker than the real gravitational waves recently discovered. In turn, this could explain why these complex fluctuations, if really existing, have not yet been discovered.

\section{DISCUSSION AND CONCLUSIONS}

Gravity-related models of spontaneous wave function collapse are not new in the literature. We mention two of them. The Diósi-Penrose model [29-32] has the same structure as the model considered here, with two important differences: (i) the noise is real and white in time, and (ii) the spatial correlation function is proportional to $G /|\mathbf{x}-\mathbf{y}|$. Although the model is certainly appealing in many ways, we see no reason why the noise correlator should have such a special form. Typically, noises have rather complicated correlation functions, which have little or no connection to the form of the interaction.

The Schrödinger-Newton equation $[32,51,52]$ descends from semiclassical gravity $[53,54]$ and contains a gravitational self-interaction term, which tends to suppress superpositions in space. However, as discussed in [55], this equation is not of the collapse-model type; in particular, it is not capable of predicting the collapse of the wave function in space with the correct quantum probabilities.

In this paper we have investigated a novel proposal, where the collapse mechanism is driven by a complex fluctuating metric, as first suggested by Adler [34]. The correlation function should have a non-negligible contribution also from relatively high frequency components $\left(\sim 10^{15} \mathrm{~Hz}\right)$, contrary to the current search for gravitational waves, which focuses on much lower frequencies.

By imposing the condition of no superluminal signaling (perturbatively up to the second order in the coupling constant $\xi$, which sets the magnitude of the gravitational noise), we derived the structure of the equation describing the evolution of the state vector [Eq. (12)]. We then proved that 
this equation defines good collapse dynamics: it collapses the state vector to the eigenstates of the preferred basis (in our case, the position basis), and it has an amplification mechanism which guarantees that, even for small $\xi$, collapse effects become relevant for macroscopic objects.

In the last section we discussed experimental bounds on the parameters of the model. Interestingly, the magnitude of the complex fluctuations needed for the collapse to be compatible with experimental data, and to guarantee the localization of macroscopic objects, can be orders of magnitude smaller than the recently discovered gravitational waves. Very weak fluctuations suffice to justify classicality as predicted by collapse models.

\section{ACKNOWLEDGMENTS}

We thank S. L. Adler for several useful comments. The authors acknowledge financial support from the University of Trieste (FRA 2016) and INFN. G. G. acknowledges financial support from ICTP Trieste.

\section{APPENDIX A: JUSTIFICATION OF THE COLLAPSE EQUATION}

We present the procedure outlined in the introduction, to justify the collapse equation. Here, to keep the notation simple, we focus on the case with only one operator $\hat{A}$ and one noise $w_{t}$. The generalization to the model described in Eq. (2) can be trivially done, since the noises are independent.

Let us consider the Hamiltonian $\hat{H}=\hat{H}_{0}+i \hbar\left(\sqrt{\lambda} \hat{A} w_{t}+\hat{O}\right)$ and, in the Itô language, the stochastic differential equation

$$
d \phi_{t}=\left[-i \hat{H}_{0} d t+\sqrt{\lambda} \hat{A} d W_{t}+\hat{O}\right] \phi_{t} ;
$$

throughout this section, we set $\hbar=1$. We will fix the form of $\hat{O}$ by requiring no superluminal signaling.

The norm of $\phi_{t}$ is not conserved. In order to write the equation for the normalized vector $\psi_{t}=\phi_{t} /\left\|\phi_{t}\right\|$, let us consider the process $N_{t}=\left\langle\phi_{t} \mid \phi_{t}\right\rangle$. Using Itô rules $\left(d N_{t}=\left\langle d \phi_{t} \mid \phi_{t}\right\rangle+\left\langle\phi_{t} \mid d \phi_{t}\right\rangle+\left\langle d \phi_{t} \mid d \phi_{t}\right\rangle\right)$, one proves that it satisfies the stochastic differential equation

$d N_{t}=\left[2 \sqrt{\lambda}\langle\hat{A}\rangle_{t} d W_{t}+\lambda\left\langle\hat{A}^{2}\right\rangle_{t} d t+\left\langle\left(\hat{O}^{\dagger}+\hat{O}\right)\right\rangle_{t} d t\right] N_{t}$,

where we have defined $\langle\hat{A}\rangle_{t}=\left\langle\phi_{t}|\hat{A}| \phi_{t}\right\rangle /\left\|\phi_{t}\right\|^{2}=$ $\left\langle\psi_{t}|\hat{A}| \psi_{t}\right\rangle$, and similarly for all other operators. From this, one can derive the equation for $N_{t}^{-1 / 2}$,

$$
\begin{aligned}
d N_{t}^{-1 / 2}= & {\left[-\sqrt{\lambda}\langle\hat{A}\rangle_{t} d W_{t}\right.} \\
& \left.+\left(\frac{3}{2} \lambda\langle\hat{A}\rangle_{t}^{2}-\frac{1}{2} \lambda\left\langle\hat{A}^{2}\right\rangle_{t}-\frac{1}{2}\left\langle\left(\hat{O}^{\dagger}+\hat{O}\right)\right\rangle_{t}\right) d t\right] N_{t}^{-1 / 2},
\end{aligned}
$$

and next the equation for $\psi_{t}=\phi_{t} N_{t}^{-1 / 2}$,

$$
\begin{aligned}
d \psi_{t}= & {\left[-i \hat{H}_{0} d t+\sqrt{\lambda}\left(\hat{A}-\langle\hat{A}\rangle_{t}\right) d W_{t}\right.} \\
& +\lambda\left(\frac{3}{2}\langle\hat{A}\rangle_{t}^{2}-\frac{1}{2}\left\langle\hat{A}^{2}\right\rangle_{t}-\hat{A}\langle\hat{A}\rangle_{t}\right) d t \\
& \left.+\left(\hat{O}-\frac{1}{2}\left\langle\left(\hat{O}^{\dagger}+\hat{O}\right)\right\rangle_{t}\right) d t\right] \psi_{t} .
\end{aligned}
$$

As we can see, the normalized vector evolves according to nonlinear stochastic dynamics. The stochastic ensemble of pure states $\rho_{t}^{W}=\left|\psi_{t}\right\rangle\left\langle\psi_{t}\right|$ obeys the following dynamics:

$$
\begin{aligned}
d \rho_{t}^{W}= & -i\left[H, \rho_{t}^{W}\right]+\lambda\left(4\langle\hat{A}\rangle_{t}^{2} \rho_{t}^{W}-\left\langle\hat{A}^{2}\right\rangle_{t} \rho_{t}^{W}-2 \hat{A}\langle\hat{A}\rangle_{t} \rho_{t}^{W}\right. \\
& \left.-2 \rho_{t}^{W} \hat{A}\langle\hat{A}\rangle_{t}-\hat{A} \rho_{t}^{W} \hat{A}\right) d t \\
& +\left(\hat{O}^{\dagger} \rho_{t}^{W}+\rho_{t}^{W} \hat{O}-\left\langle\left(\hat{O}^{\dagger}+\hat{O}\right)\right\rangle_{t} \rho_{t}^{W}\right) d t \\
& +(\text { extra terms }) d W_{t} .
\end{aligned}
$$

When taking the expectation value to compute the dynamics for the density matrix $\rho_{t}=\mathbb{E}\left[\rho_{t}^{W}\right]$, the "extra terms" average to 0 , while the remaining terms generate a nonlinear evolution for the ensemble. This can be avoided by choosing $O=-(\lambda / 2) \hat{A}^{2}+2 \lambda\left(\hat{A}-\langle\hat{A}\rangle_{t}\right)\langle\hat{A}\rangle_{t}$, in which case all nonlinear terms cancel, and the equation for $\rho_{t}$ becomes of the Lindblad type,

$$
\frac{d}{d t} \rho_{t}=-i\left[\hat{H}_{0}, \rho_{t}\right]-\frac{\lambda}{2}\left[\hat{A},\left[\hat{A}, \rho_{t}\right]\right] ;
$$

in turn, Eq. (A1) reduces to Eq. (1). This completes the argument.

\section{APPENDIX B: NONRELATIVISTIC COUPLING BETWEEN A GRAVITATIONAL BACKGROUND AND THE LOCAL MASS DENSITY}

The action of a matter field in curved space is described by

$$
S=\int d^{4} x \sqrt{-g} \mathcal{L}_{m},
$$

where $\mathcal{L}_{m}$ is the matter Lagrangian, $g_{\mu \nu}$ is the metric tensor, and $\sqrt{-g}=\sqrt{-\operatorname{det}\left[g_{\mu \nu}\right]}$. We consider a perturbation $h_{\mu \nu}$ around the flat metric $\eta_{\mu \nu}$, and we Taylor expand the action around it,

$$
S=\int d^{4} x\left[\mathcal{L}_{m}^{(0)}+\left.\frac{1}{\sqrt{-\eta}} \frac{\partial(\sqrt{-g} \mathcal{L})}{\partial g_{\mu \nu}}\right|_{\eta_{\mu \nu}} h_{\mu \nu}\right]+\mathcal{O}\left(h^{\mu \nu} h^{\delta \sigma}\right) ;
$$

the apex (0) denotes the quantities in the flat spacetime $\eta_{\mu \nu}$. The stress energy tensor associated with the Lagrangian $\mathcal{L}_{m}$ is defined as follows [56]: 


$$
T^{\mu \nu}=\frac{-2}{\sqrt{-g}} \frac{\partial\left(\sqrt{-g} \mathcal{L}_{m}\right)}{\partial g_{\mu \nu}},
$$

and Eq. (B2) can be rewritten in the form

$$
S=\int d^{4} x\left[\mathcal{L}_{m}^{(0)}-\frac{1}{2} h^{\mu \nu} T_{\mu \nu}^{(0)}\right]
$$

where from now on we neglect higher-order terms. In the weak field limit, gravity couples to matter through the stress energy tensor.

We now derive the nonrelativistic limit for a KleinGordon Lagrangian,

$$
\mathcal{L}_{m}^{(0)}=\frac{-\hbar^{2}}{2 m}\left(\eta^{\mu \nu} \partial_{\mu} \psi^{*} \partial_{\nu} \psi-\frac{(m c)^{2}}{\hbar^{2}} \psi^{*} \psi\right) .
$$

The interacting Lagrangian becomes

$$
\begin{aligned}
\mathcal{L}_{\text {int }}^{(0)} & =-\frac{1}{2} h^{\mu \nu} T_{\mu \nu}^{(0)} \\
& =\frac{\hbar}{2 m}\left(\partial_{\mu} \psi^{*} \partial_{\nu} \psi\left(h^{\mu \nu}-h_{\rho}^{\rho} \eta^{\mu \nu}\right)-h_{\rho}^{\rho} \frac{m c^{2}}{2 \hbar^{2}} \psi^{*} \psi\right) .
\end{aligned}
$$

The nonrelativistic limit can be obtained by rewriting the relativistic wave function as follows:

$$
\psi(x)=e^{\frac{i}{\hbar} m c x_{0}} \varphi(x),
$$

and assuming that the following relation holds:

$$
\left|\frac{m c}{\hbar} \varphi\right| \gg\left|i \partial_{\mu} \varphi\right|,
$$

meaning that the rest energy associated to the field $\varphi$ is much bigger than the momentum energy. Inserting Eq. (B7) into Eqs. (B5) and (B6), one obtains

$$
\begin{aligned}
\mathcal{L}_{m}^{(0)}= & -\frac{\hbar}{2 m}\left[\partial_{0} \varphi^{*} \partial_{0} \varphi\right. \\
& \left.+i \frac{m c}{\hbar}\left(\varphi^{*} \partial_{0} \varphi-\left(\partial_{0} \varphi^{*}\right) \varphi\right)+\partial_{i} \varphi^{*} \partial_{i} \varphi\right]
\end{aligned}
$$

and

$$
\begin{aligned}
\mathcal{L}_{\text {int }}^{(0)}= & -\frac{1}{2} h^{\mu \nu} T_{\mu \nu}^{(0)} \\
= & -\frac{\hbar^{2}}{2 m}\left[h^{00}\left(\partial_{0}-i \frac{m c}{\hbar}\right) \varphi^{*}\left(\partial_{0}+i \frac{m c}{\hbar}\right) \varphi\right. \\
& -h^{0 i}\left[\left(\partial_{0}-i \frac{m c}{\hbar}\right) \varphi^{*} \partial_{i} \varphi+\partial_{i} \varphi^{*}\left(\partial_{0}+i \frac{m c}{\hbar}\right) \varphi\right] \\
& \left.+\left(h^{i j}-h_{\rho}^{\rho} \eta^{i j}\right) \partial_{i} \varphi^{*} \partial_{i} \varphi\right] .
\end{aligned}
$$

Under the assumption in (B8), we arrive at the symmetrized free Schrödinger Lagrangian $\left(x_{0}=c t\right)$,

$$
\mathcal{L}_{m}^{(0)} \simeq \frac{i \hbar}{2}\left(\varphi^{*} \partial_{t} \varphi-\partial_{t}\left(\varphi^{*}\right) \varphi\right)+\frac{\hbar^{2}}{2 m} \partial_{i} \varphi^{*} \partial^{i} \varphi,
$$

and the interaction Lagrangian

$$
\mathcal{L}_{\mathrm{int}}^{(0)}=-\frac{m c^{2}}{2} h^{00} \varphi^{*} \varphi
$$

The conjugate momenta associated with the total Lagrangian $\mathcal{L}=\mathcal{L}_{m}^{(0)}+\mathcal{L}_{\text {int }}^{(0)}$ are

$$
\begin{gathered}
\pi=\frac{\partial \mathcal{L}}{\partial\left(\partial_{t} \varphi\right)}=\frac{i \hbar}{2} \varphi^{*}, \\
\pi^{*}=\frac{\partial \mathcal{L}}{\partial\left(\partial_{t} \varphi^{*}\right)}=-\frac{i \hbar}{2} \varphi,
\end{gathered}
$$

and the Hamiltonian density is

$$
\begin{aligned}
\mathcal{H}(x) & =\pi \partial_{t} \varphi^{*}+\pi^{*} \partial_{t} \varphi-\mathcal{L} \\
& =\frac{\hbar^{2}}{2 m} \partial_{i} \varphi^{*} \partial^{i} \varphi+\frac{m c^{2}}{2} h^{00} \varphi^{*} \varphi,
\end{aligned}
$$

leading, after integration by parts, to the Hamiltonian

$H=\int d^{3} x \varphi^{*}(\mathbf{x}, t)\left(-\frac{\hbar^{2}}{2 m} \partial_{i} \partial^{i}+\frac{m c^{2}}{2} h^{00}(\mathbf{x}, t)\right) \varphi(\mathbf{x}, t)$.

Promoting the field $\varphi(x)\left(\varphi^{*}(x)\right)$ and its conjugate momenta $\pi(x)\left(\pi(x)^{*}\right)$ to operators

$$
\begin{aligned}
\varphi(\mathbf{x}, t) & \rightarrow \hat{\varphi}(\mathbf{x}, t), \\
\pi(\mathbf{x}, t) & \rightarrow \hat{\pi}(\mathbf{x}, t)
\end{aligned}
$$

and imposing the canonical quantization rule, i.e.,

$$
\begin{aligned}
{[\hat{\varphi}(\mathbf{x}, t), \hat{\pi}(\mathbf{x}, t)] } & =\left[\hat{\varphi}^{\dagger}(\mathbf{x}, t), \hat{\pi}^{\dagger}(\mathbf{x}, t)\right] \\
& =i \hbar \delta(\mathbf{x}-\mathbf{y})
\end{aligned}
$$

one obtains the Hamiltonian

$$
\hat{H}=\int d^{3} x \hat{\varphi}^{\dagger}(\mathbf{x}) H_{1}(x) \hat{\varphi}(\mathbf{x}),
$$

where

$$
H_{1}(\mathbf{x}, t)=-\frac{\hbar^{2}}{2 m} \partial_{i} \partial^{i}+\frac{m c^{2}}{2} h^{00}(\mathbf{x}, t)
$$

is the single-particle Hamiltonian expressed in the position basis. 


\section{APPENDIX C: STOCHASTIC SCHRÖDINGER EQUATION AND NON-FASTER-THAN-LIGHT SIGNALING}

The calculations leading to the main result of this paper are rather involved. In this appendix we provide the technical details of the derivation of Eq. (12) and Eq. (13). The perturbation expansion obtained by combining Eq. (8) with Eq. (9) and Eq. (10) gives the following system of equations:

$$
\begin{aligned}
i \hbar \partial_{t} \rho_{0, t}^{h}= & \hat{H}_{0} \rho_{0, t}^{h}-\text { H.c. } \\
i \hbar \partial_{t} \rho_{1, t}^{h}= & \hat{H}_{0} \rho_{1, t}^{h}+\left(\sum_{i=1}^{N}\left(\hat{A}_{i} h_{i}(t)-i\left\langle A_{i}\right\rangle_{t}^{0} h_{i}^{\mathrm{I}}(t)+\hat{O}_{1}+\frac{1}{2}\left\langle\hat{O}_{1}-\hat{O}_{1}^{\dagger}\right\rangle_{t}^{0}\right) \rho_{0, t}^{h}-\right.\text { H.c. } \\
i \hbar \partial_{t} \rho_{2, t}^{h}= & \hat{H}_{0} \rho_{2, t}^{h}+\left(\sum_{i=1}^{N}\left(\hat{A}_{i} h_{i}(t)-i\left\langle A_{i}\right\rangle_{t}^{0} h_{i}^{\mathrm{I}}(t)+\hat{O}_{1}-\frac{1}{2}\left\langle\hat{O}_{1}-\hat{O}_{1}^{\dagger}\right\rangle_{t}^{0}\right) \rho_{1, t}^{h}\right. \\
& -\left(\sum_{i=1}^{N} i\left\langle A_{i}\right\rangle_{t}^{1} h_{i}^{\mathrm{I}}(t)-\hat{O}_{2}+\frac{1}{2}\left\langle\hat{O}_{1}-\hat{O}_{1}^{\dagger}\right\rangle_{t}^{1}+\frac{1}{2}\left\langle\hat{O}_{2}-\hat{O}_{2}^{\dagger}\right\rangle_{t}^{0}\right) \rho_{0, t}^{h}-\text { H.c. }
\end{aligned}
$$

where $\langle A\rangle_{t}^{n}=\operatorname{Tr}\left(\hat{A} \rho_{n, t}^{h}\right)$, and similarly for the other operators. We can formally solve the above system of equations as follows:

$$
\begin{aligned}
\rho_{0, t}^{h}= & e^{i \hat{H}_{0} t} \rho_{0} e^{-i \hat{H}_{0} t} \\
\rho_{1, t}^{h}= & -\frac{i}{\hbar} \sum_{i=1}^{N} \int_{0}^{t} d \tau\left(\hat{A}_{i}(\tau-t) h_{i}(\tau)-i\left\langle A_{i}\right\rangle_{\tau}^{0} h_{i}^{\mathrm{I}}(\tau)+\hat{O}_{1}(\tau-t)-\frac{1}{2}\left\langle O_{1}-O_{1}^{\dagger}\right\rangle_{\tau}^{0}\right) \rho_{0, t}^{h}+\text { H.c. } \\
\rho_{2, t}^{h}= & -\frac{i}{\hbar} \sum_{i=1}^{N} \int_{0}^{t} d \tau\left(\hat{A}_{i}(\tau-t) h_{i}(\tau)-i\left\langle A_{i}\right\rangle_{\tau}^{0} h_{i}^{\mathrm{I}}(\tau)+\hat{O}_{1}(\tau-t)-\frac{1}{2}\left\langle O_{1}-O_{1}^{\dagger}\right\rangle_{\tau}^{0}\right) e^{i \hat{H}(t-\tau)} \rho_{1, \tau}^{h} e^{-i \hat{H}(t-\tau)} \\
& -\frac{i}{\hbar} \sum_{i=1}^{N} \int_{0}^{t} d \tau\left(i\left\langle A_{i}\right\rangle_{\tau}^{1} h_{i}^{\mathrm{I}}(\tau)-\hat{O}_{2}(\tau-t)+\frac{1}{2}\left\langle\hat{O}_{1}-\hat{O}_{1}^{\dagger}\right\rangle_{\tau}^{1}+\frac{1}{2}\left\langle O_{2}-O_{2}^{\dagger}\right\rangle_{\tau}^{0}\right) \rho_{0, t}^{h}+\text { H.c., }
\end{aligned}
$$

where $\hat{A}_{i}(t)$ is the operator $\hat{A}_{i}$ in the interaction picture at time $t$,

$$
\hat{A}_{i}(t)=e^{\frac{i}{\hbar} \hat{H}_{0} t} \hat{A}_{i} e^{-\frac{i}{\hbar} \hat{H}_{0} t}
$$

and similarly for the operator $\hat{O}$. Now we are in the position to compute a closed equation for the averaged density matrix $\mathbb{E}\left[\rho_{t}^{h}\right]$. We plug the solutions in Eq. (C2) into Eq. (9); in this way, the stochasticity is entirely contained in polynomials of $h$, whose correlations are known. We can then explicitly compute the stochastic average of each term. Collecting all pieces together, we arrive at the following perturbative equations for the ensemble, which are valid up to order $\xi^{2}$ :

$$
\begin{aligned}
i \hbar \partial_{t} \rho_{0, t}= & \hat{H}_{0} \rho_{0, t}-\text { H.c. } \\
i \hbar \partial_{t} \rho_{1, t}= & \hat{O}_{1} \rho_{0, t}+\frac{1}{2}\left\langle O_{1}-O_{1}^{\dagger}\right\rangle_{t}^{0} \rho_{0, t}-\text { H.c. } \\
i \hbar \partial_{t} \rho_{2, t}= & -\frac{i}{\hbar} \sum_{i, j=1}^{N} \int_{0}^{t} d \tau S_{i j}(t, \tau)\left(\hat{A}_{i}-\left\langle A_{i}\right\rangle_{t}^{0}\right)\left(\hat{A}_{j}(\tau-t)-\left\langle A_{j}(\tau-t)\right\rangle_{t}^{0}\right) \rho_{0, t} \\
& +\frac{i}{\hbar} \sum_{i, j=1}^{N} \int_{0}^{t} d \tau D_{i j}(t, \tau)\left(\hat{A}_{j}(\tau-t)-\left\langle A_{j}(\tau-t)\right\rangle_{t}^{0}\right) \rho_{0, t}(t, \tau)\left(\hat{A}_{i}-\left\langle A_{i}\right\rangle_{t}^{0}\right) \\
& +\frac{i}{\hbar} \sum_{i, j=1}^{N} \int_{0}^{t} d \tau\left(S_{i j}(t, \tau)-D_{i j}(t, \tau)\right)\left\langle A_{i}\left(A_{j}(\tau-t)-\left\langle A_{j}(\tau-t)\right\rangle_{t}^{0}\right)\right\rangle_{t}^{0} \rho_{0, t} \\
& +\hat{O}_{2}+\frac{1}{2}\left\langle O_{2}-O_{2}^{\dagger}\right\rangle_{t}^{0} \rho_{0, t}-\text { H.c. }
\end{aligned}
$$


The above equations are again nonlinear. The nonlinear terms can be removed by choosing

$$
\begin{aligned}
\hat{O}_{1}= & 0 \\
\hat{O}_{2}= & +\frac{i}{\hbar} \sum_{i, j=1}^{N} \int_{0}^{t} d \tau\left(S_{i j}(t, \tau)-D_{i j}(t, \tau)\right) \hat{A}_{i}\left(\hat{A}_{j}(t-\tau)-\left\langle A_{j}(t-\tau)\right\rangle_{t}^{0}\right) \\
& -\frac{i}{\hbar} \sum_{i, j=1}^{N} \int_{0}^{t} d \tau\left(S_{i j}(t, \tau)-D_{i j}^{*}(t, \tau)\right)\left\langle A_{i}\right\rangle_{t}^{0} \hat{A}_{j}(\tau-t) .
\end{aligned}
$$

Substituting this expression into Eq. (C4) and resumming the Taylor series, one arrives at

$$
\partial_{t} \rho_{t}=-\frac{i}{\hbar} \hat{H}_{0} \rho_{t}-\frac{\xi^{2}}{\hbar^{2}} \int_{0}^{t} d \tau \sum_{i, j=1}^{N} D_{i j}(t, \tau)\left(\hat{A}_{i} \hat{A}_{j}(\tau-t) \rho_{t}-\hat{A}_{j}(\tau-t) \rho_{t} \hat{A}_{i}\right)+\mathcal{O}\left(\xi^{3}\right)+\text { H.c. }
$$

or equivalently,

$$
\begin{aligned}
\partial_{t} \rho_{t}= & -\frac{i}{\hbar}\left[\hat{H}_{0}, \rho_{t}\right]-\frac{\xi^{2}}{\hbar^{2}} \sum_{i, j=1}^{N} \int_{0}^{t} d \tau D_{i j}^{\mathrm{R}}(t, \tau)\left[\hat{A}_{i},\left[\hat{A}_{j}(\tau-t), \rho_{t}\right]\right] \\
& -\frac{i \xi^{2}}{\hbar^{2}} \sum_{i, j=1}^{N} \int_{0}^{t} d \tau D_{i j}^{\mathrm{I}}(t, \tau)\left[\hat{A}_{i},\left\{\hat{A}_{j}(\tau-t), \rho_{t}\right\}\right]+\mathcal{O}\left(\xi^{3}\right)
\end{aligned}
$$

\section{APPENDIX D: RELATION TO THE HPZ MASTER EQUATION}

Let us start with the center of mass master equation given by Eq. (32) with the free particle Hamiltonian $\hat{H}_{0}^{\mathrm{CM}}=\frac{\hat{\boldsymbol{P}}^{2}}{2 m}$, where $\hat{\boldsymbol{P}}$ is the center of mass momentum operator. We make two assumptions regarding the noise correlation functions $\tilde{D}^{\mathrm{R}}(\mathbf{Q}, s), \tilde{D}^{\mathrm{I}}(\mathbf{Q}, s)$ and the center of mass state $\rho_{t}$. Loosely speaking, we restrict to a nearly Markovian regime and assume that the exchanged momentum between noise and system is small. Mathematically, we give the sufficient conditions to expand the operators to quadratic order, i.e., to order $\mathcal{O}\left(\hat{X}^{2}\right), \mathcal{O}\left(\hat{P}^{2}\right), \mathcal{O}(\hat{X} \hat{P})$ :

(a) The noise correlation times are small and the state $\rho_{t}$ is such that

$$
e^{\frac{i}{\hbar} \hat{H}_{0}^{\mathrm{CM}} s} \approx 1+\frac{i}{\hbar} \hat{H}_{0}^{\mathrm{CM}} s=1+\frac{i}{\hbar} \frac{\hat{P}^{2}}{2 m} s
$$

(b) The noise momentum correlations are small and the state $\rho_{t}$ is such that

$$
e^{\frac{i}{\hbar} \mathbf{Q} \cdot \hat{\boldsymbol{X}}} \approx 1+\frac{i}{\hbar} \mathbf{Q} \cdot \hat{\boldsymbol{X}}-\frac{1}{\hbar^{2}}(\mathbf{Q} \cdot \hat{\boldsymbol{X}})^{2}
$$

Moreover, the noise momentum correlations depend only on the modulus $Q=|\mathbf{Q}|$,

$$
\begin{gathered}
\tilde{D}^{\mathrm{R}}(\mathbf{Q}, s)=\tilde{D}^{\mathrm{R}}(Q, s), \\
\tilde{D}^{\mathrm{I}}(\mathbf{Q}, s)=\tilde{D}^{\mathrm{I}}(Q, s),
\end{gathered}
$$

which is equivalent, as follows from Eq. (25), to assuming a noise correlation isotropic in space $D^{\mathrm{R}}(\mathbf{r}, s)=D^{\mathrm{R}}(r, s)$ and $D^{\mathrm{I}}(\mathbf{r}, s)=D^{\mathrm{I}}(r, s)$ with $r=|\mathbf{r}|$. (c) The noise correlation time $\tau_{0}$ is small with respect to the evolution time $t$.

Applying the above assumptions (a), (b), and (c), using the formula in Eq. (35) for the amplification factors and the identity

$$
\begin{aligned}
& \int d \mathbf{Q} f(Q)(\mathbf{Q} \cdot \mathbf{X})(\mathbf{Q} \cdot \mathbf{Y}) \\
& =\left(\int_{0}^{\infty} d Q f(Q) Q^{4}\right) \frac{4 \pi}{3} \mathbf{X} \cdot \mathbf{Y},
\end{aligned}
$$

where $f(Q)$ denotes a generic function, we can perform the $\mathbf{Q}$ integration in Eq. (32). After some algebra we obtain the simplified master equation

$$
\begin{aligned}
\frac{d \rho_{t}^{\mathrm{CM}}}{d t}= & -\frac{i}{\hbar} \sum_{j=1}^{3}\left[\frac{\hat{P}_{j}^{2}}{2 m}, \rho_{t}\right]-\eta \frac{A^{\mathrm{R}}\left(r_{C}^{\mathrm{R}}\right)}{m_{0}^{2}} \sum_{j=1}^{3}\left[\hat{X}_{j},\left[\hat{X}_{j}, \rho_{t}\right]\right] \\
& +\Pi \frac{A^{\mathrm{R}}\left(r_{C}^{\mathrm{R}}\right)}{m_{0}^{2}} \sum_{j=1}^{3}\left[\hat{X}_{j},\left[\hat{P}_{j} / m, \hat{\rho}\right]\right] \\
& -i \Upsilon \frac{A^{\mathrm{I}}\left(r_{C}^{\mathrm{I}}\right)}{m_{0}^{2}} \sum_{j=1}^{3}\left[\hat{X}_{j},\left\{\hat{P}_{j} / m, \rho_{t}\right\}\right],
\end{aligned}
$$

where 


$$
\begin{gathered}
\eta=\frac{m_{0}^{2} c^{4} \xi^{2}}{6 \pi^{2} \hbar^{7}} \int_{0}^{\infty} d Q \int_{0}^{\infty} d \tau \tilde{D}^{\mathrm{R}}(Q, \tau) Q^{4}, \\
\Pi=\frac{m_{0}^{2} c^{4} \xi^{2}}{6 \pi^{2} \hbar^{7}} \int_{0}^{\infty} d Q \int_{0}^{\infty} d \tau \tau \tilde{D}^{\mathrm{R}}(Q, \tau) Q^{4}, \\
\Upsilon=-\frac{m_{0}^{2} c^{4} \xi^{2}}{6 \pi^{2} \hbar^{7}} \int_{0}^{\infty} d Q \int_{0}^{\infty} d \tau \tau \tilde{D}^{\mathrm{I}}(Q, \tau) Q^{4}
\end{gathered}
$$

are three phenomenological parameters [given assumption (c), these do not depend on $t]$, while $A^{\mathrm{R}}\left(r_{C}^{\mathrm{R}}\right) / m_{0}^{2}$ and $A^{\mathrm{I}}\left(r_{C}^{\mathrm{I}}\right) / m_{0}^{2}$ are dimensionless amplification factors, related to the size and shape of the composite object as well as to the noise spatial correlation cutoffs $r_{C}^{\mathrm{R}}$ and $r_{C}^{\mathrm{I}}$ (see Sec. IV).

Equation (D5) has the same structure as the HPZ master equation [57], except for the absence of the HPZ term that breaks translational invariance. The reason why the HPZ master equation breaks translational invariance lies in its founding assumption: a particle in a harmonic potential coupled to a bath of oscillators. In our case, loosely speaking, the external oscillators correspond to the complex noise, while the harmonic potential, which explicitly breaks translational invariance, is absent. Our noise does not break translational invariance, as we have assumed explicitly that the correlation function is translationally invariant (see Sec. IV).
[1] A. J. Leggett, Macroscopic quantum systems and the quantum theory of measurement, Prog. Theor. Phys. Suppl. 69, 80 (1980).

[2] R. Drever, in Quantum Optics, Experimental Gravity, and Measurement Theory (Plenum, New York, 1983).

[3] R. Penrose and C. J. Isham, Quantum Concepts in Space and Time (Oxford University Press, New York, 1986).

[4] T. D. Clark, in Quantum Implications: Essays in Honour of David Bohm, edited by B. J. Hiley and F. D. Peat (Routledge, New York, 1987).

[5] S. Weinberg, Precision Tests of Quantum Mechanics, Phys. Rev. Lett. 62, 485 (1989).

[6] S. L. Adler, Quantum Theory as an Emergent Phenomenon: The Statistical Mechanics of Matrix Models as the Precursor of Quantum Field Theory (Cambridge University Press, Cambridge, England, 2004), p. 165.

[7] J. S. Bell, Speakable and Unspeakable in Quantum Mechanics: Collected Papers on Quantum Philosophy (Cambridge University Press, Cambridge, England, 2004).

[8] G. C. Ghirardi, A. Rimini, and T. Weber, Unified dynamics for microscopic and macroscopic systems, Phys. Rev. D 34, 470 (1986).

[9] G. C. Ghirardi, P. Pearle, and A. Rimini, Markov processes in Hilbert space and continuous spontaneous localization of systems of identical particles, Phys. Rev. A 42, 78 (1990).

[10] A. Bassi and G. C. Ghirardi, Dynamical reduction models, Phys. Rep. 379, 257 (2003).

[11] A. Bassi, K. Lochan, S. Satin, T. P. Singh, and H. Ulbricht, Models of wave-function collapse, underlying theories, and experimental tests, Rev. Mod. Phys. 85, 471 (2013).

[12] S. K. Modak, L. Ortíz, I. Peña, and D. Sudarsky, Nonparadoxical loss of information in black hole evaporation in a quantum collapse model, Phys. Rev. D 91, 124009 (2015).

[13] S. K. Modak, L. Ortíz, I. Peña, and D. Sudarsky, Black hole evaporation: Information loss but no paradox, Gen. Relativ. Gravit. 47, 120 (2015).

[14] D. Bedingham, S. K. Modak, and D. Sudarsky, Relativistic collapse dynamics and black hole information loss, Phys. Rev. D 94, 045009 (2016).
[15] S. L. Adler, D. C. Brody, T. A. Brun, and L. P. Hughston, Martingale models for quantum state reduction, J. Phys. A 34, 8795 (2001).

[16] A possible hint for this anzatz can be found in the works by M. B. Mensky et al. [17-20]. In particular, in [19] it is shown how the presence of a continuous measurement (whose effects are expected to be similar to those of a continuous collapse) can be described effectively by adding an anti-Hermitian term to the Hamiltonian of the system.

[17] M. B. Mensky, Quantum restrictions on the measurement of the parameters of motion of a macroscopic oscillator, Sov. Phys. JETP 50, 667 (1979).

[18] M. B. Mensky, Quantum restrictions for continuous observation of an oscillator, Phys. Rev. D 20, 384 (1979).

[19] G. A. Golubtsova and M. B. Mensky, Quantum nondemolition measurements from path integral, Int. J. Mod. Phys. A 04, 2733 (1989).

[20] M. B. Mensky, Continuous Quantum Measurements and Path Integrals (IOP Publishing, Philadelphia, 1993).

[21] N. Gisin, Stochastic quantum dynamics and relativity, Helv. Phys. Acta 62, 363 (1989).

[22] G. Lindblad, On the generators of quantum dynamical semigroups, Commun. Math. Phys. 48, 119 (1976).

[23] V. Gorini, A. Kossakowski, and E. C. G. Sudarshan, Completely positive dynamical semigroups of n-level systems, J. Math. Phys. (N.Y.) 17, 821 (1976).

[24] H.-P. Breuer and F. Petruccione, The Theory of Open Quantum Systems (Oxford University Press, New York, 2002).

[25] F. Karolyhazy, Gravitation and quantum mechanics of macroscopic objects, Nuovo Cimento A 42, 390 (1966).

[26] F. Karolyhazy, J. A. Frenkel, and B. Lukács, in Quantum Concepts in Space and Time, edited by R. Penrose and Chris J. Isham (Clarendon Press, Oxford, 1986).

[27] F. Karolyhazy, in Sixty-Two Years of Uncertainty, edited by A. I. Miller (Plenum, New York, 1990).

[28] A. Frenkel, A tentative expression of the Karolyhazy uncertainty of the space-time structure through vacuum spreads in quantum gravity, Found. Phys. 32, 751 (2002). 
[29] L. Diósi, A universal master equation for the gravitational violation of quantum mechanics, Phys. Lett. A 120, 377 (1987).

[30] L. Diósi, Models for universal reduction of macroscopic quantum fluctuations, Phys. Rev. A 40, 1165 (1989).

[31] L. Diósi, Notes on certain newton gravity mechanisms of wavefunction localization and decoherence, J. Phys. A 40, 2989 (2007).

[32] R. Penrose, On gravity's role in quantum state reduction, Gen. Relativ. Gravit. 28, 581 (1996).

[33] Here the discrete label " $j$ " is replaced by the continuous label $\mathbf{x}$ representing the space points. Accordingly, the discrete sums over $j$ become an integral over $\mathbf{x}$.

[34] S. L. Adler, Gravitation and the noise needed in objective reduction models, in Quantum Nonlocality and Reality: 50 Years of Bell's Theorem, edited by M. Bell and S. Gao (Cambridge University Press, Cambridge, England 2016).

[35] K. Krasnov, Gr uniqueness and deformations, J. High Energy Phys. 10 (2015) 037.

[36] S. L. Adler and A. Bassi, Collapse models with non-white noises, J. Phys. A 40, 15083 (2007).

[37] In the expansion of $\hat{O}$ we do not include a zero-order term, i.e., one independent from $\xi$. This is because $\hat{O}$ is introduced to add corrective terms due to the presence of the noise terms, and therefore is expected to be zero in the limit $\xi \rightarrow 0$.

[38] Note that the correlator $S_{i j}(t, \tau)$ does not appear.

[39] See p. 9 in [36].

[40] Here again the discrete label $i$ is replaced by the continuous parameter $\mathbf{x}$

[41] M. Toroš and A. Bassi, Bounds on collapse models from matter-wave interferometry: Calculational details, arXiv: 1601.02931

[42] S. L Adler, Lower and upper bounds on CSL parameters from latent image formation and IGM heating, J. Phys. A 40, 2935 (2007).

[43] M. Carlesso, A. Bassi, P. Falferi, and A. Vinante, Experimental bounds on collapse models from gravitational wave detectors, Phys. Rev. D 94, 124036 (2016).
[44] C. Curceanu, B. C. Hiesmayr, and K. Piscicchia, X-rays help to unfuzzy the concept of measurement, Adv. Phys. 4, 263 (2015).

[45] M. Bilardello, S. Donadi, A. Vinante, and A. Bassi, Bounds on collapse models from cold-atom experiments, Physica A (Amsterdam) 462A, 764 (2016).

[46] M. Toroš and A. Bassi, Bounds on collapse models from matter-wave interferometry, arXiv:1601.03672.

[47] M. Bahrami, M. Paternostro, A. Bassi, and H. Ulbricht, Proposal for a Noninterferometric Test of Collapse Models in Optomechanical Systems, Phys. Rev. Lett. 112, 210404 (2014).

[48] S. Nimmrichter, K. Hornberger, and K. Hammerer, Optomechanical Sensing of Spontaneous Wave-Function Collapse, Phys. Rev. Lett. 113, 020405 (2014).

[49] L. Diósi, Testing Spontaneous Wave-Function Collapse Models on Classical Mechanical Oscillators, Phys. Rev. Lett. 114, 050403 (2015).

[50] B. P. Abbott et al., Observation of Gravitational Waves from a Binary Black Hole Merger, Phys. Rev. Lett. 116, 061102 (2016).

[51] L. Diósi, Gravitation and quantum-mechanical localization of macro-objects, Phys. Lett. 105A, 199 (1984).

[52] S. Carlip, Is quantum gravity necessary?, Classical Quantum Gravity 25, 154010 (2008).

[53] C. Møller, Les théories relativistes de la gravitation, Colloq. Int. CNRS 91, 154010 (1962).

[54] L. Rosenfeld, On quantization of fields, Nucl. Phys. 40, 353 (1963).

[55] M. Bahrami, A. Großardt, S. Donadi, and A. Bassi, The Schrödinger-Newton equation and its foundations, New J. Phys. 16, 115007 (2014).

[56] S. M. Carroll, Spacetime and Geometry: An Introduction to General Relativity, Vol. 1 (Pearson, London, England, 2004).

[57] B. L. Hu, J. P. Paz, and Y. Zhang, Quantum Brownian motion in a general environment: Exact master equation with nonlocal dissipation and colored noise, Phys. Rev. D 45, 2843 (1992). 